\title{
Primary pericardial angiosarcoma: case report and review of treatment options
}

\author{
Udit Yadav ${ }^{1}$ and Ankit Mangla ${ }^{2}$ \\ ${ }^{1}$ Department of Medicine, Division of Hematology and Oncology, John H Stroger, Jr Hospital of Cook County, Chicago, IL, USA \\ ${ }^{2}$ Department of Hematology and Oncology, Case Western University School of Medicine, Cleveland, OH, USA
}

\begin{abstract}
A primary cardiac angiosarcoma is a rare type of soft-tissue sarcoma with a high mortality rate. This report describes a young woman who presented with chest pain and worsening shortness of breath over the course of a year. She was diagnosed with and treated for latent tuberculosis and autoimmune pericarditis over the last year, however, her condition kept worsening. Further workup revealed a large pericardial and right atrial mass associated with multiple lung nodules. The biopsy from the lung mass showed angiosarcoma, and she was diagnosed with primary metastatic angiosarcoma of the pericardium. She was treated with doxorubicin and Ifosfamide (AIM-75 regimen), which led to a partial response. However, soon after completion of six cycles, the tumour progressed rapidly, leading to cardio-respiratory failure. In this report, we will discuss the clinical challenges and treatment options (surgical and medical) that are available for treating patients with angiosarcoma of the heart.
\end{abstract}

Keywords: cardiac tumour, angiosarcoma

\section{Introduction}

Primary cardiac tumours (PCT) are rare tumours, with an autopsy prevalence estimated at $0.001 \%$ to $0.03 \%$ [1]. A metastatic deposit to the heart from a distant primary is $20-30$ times more common than a primary tumour of the heart [2]. A quarter of all PCT are malignant, and 50\%-75\% of all malignant PCT are angiosarcomas [1, 3-5]. Surgical resection of the tumour is the treatment of choice; however, achieving a microscopically negative surgical margin (RO resection) is a challenge due to the complex anatomy and proximity with critical structures [6, 7]. Currently, there is no established role of neoadjuvant and adjuvant chemotherapy in patients with localised PCT. Patients who present with metastatic disease have a dismal prognosis with a median life span of less than 6 months [8]. Identifying patients with PCT is a clinical challenge, and imaging and histopathology are critical to establishing a diagnosis. In this report, we will discuss the clinical challenges in a patient with metastatic primary cardiac angiosarcoma. We will also review the literature regarding the treatment of this rare yet fatal cancer.
Correspondence to: Ankit Mangla Email: axm1297@case.edu

ecancer 2020, 14:1056

https://doi.org/10.3332/ecancer.2020.1056

Published: $15 / 06 / 2020$

Received: 03/03/2020

Publication costs for this article were supported by ecancer (UK Charity number 1176307).

Copyright: (c) the authors; licensee ecancermedicalscience. This is an Open Access article distributed under the terms of the Creative Commons Attribution License (http:// creativecommons.org/licenses/by/3.0), which permits unrestricted use, distribution, and reproduction in any medium, provided the original work is properly cited. 


\section{Case presentation}

A 44-year-old woman presented to the emergency room at our institution with chest pain and shortness of breath. She had no significant past medical history or family history. She denied any history of smoking, alcohol consumption or illicit drug use. A year prior to this visit, she had presented to another hospital with similar complaints. The workup at that time demonstrated pericardial effusion, and the cytology of the pericardial fluid was suggestive of exudative effusion. At that visit, she was diagnosed with latent tuberculosis after a positive tuberculin skin test and received treatment with isoniazid for 6 months. However, her symptoms kept worsening over 6 months, and she presented to our hospital with severe shortness of breath. She gave a history of dyspnea on exertion, occasional squeezing non-radiating chest pain and palpitations. The review of systems was notable for anorexia and significant weight loss over the last six months. At the time of the presentation, she was in significant respiratory distress. The systemic exam was significant for jugular venous distention, bilateral pitting edema of lower extremities, pallor, and use of accessory muscles of respiration. The cardiovascular exam was significant for soft first heart sound and non-palpable apex. The rest of the systemic examination was unremarkable.

Labs at presentation were remarkable for normochromic normocytic anaemia ( $\mathrm{Hb}-11.4 \mathrm{gm} / \mathrm{dL}$ ) only. The comprehensive metabolic panel, troponin-I and B-natriuretic peptide were normal at presentation. The bedside echocardiogram revealed an ejection fraction of 55\%-60\%, normal valvular structures, and a moderate, free-flowing, concentric pericardial effusion without tamponade physiology. CT scan of the chest revealed pericardial effusion with a possible pericardial sac mass and multiple bilateral hemorrhagic pulmonary nodules. Cardiac CT showed a highly vascular, right atrial wall soft tissue mass involving the adjacent pericardium associated with large pericardial effusion (Figure 1). CT guided biopsy of the mass showed a tumour with spindle and epithelioid cells, prominent nucleoli and irregular vascular channels (Figure 2). CD31 and CD34 stains were positive in the tumour cells and highlighted the vasculature. The tumour cells were negative for keratin AE1/ AE3, EMA, BerEP4, CK7, TTF-1, calretinin, CK5/6, MART-1, HMB45 and S-100.

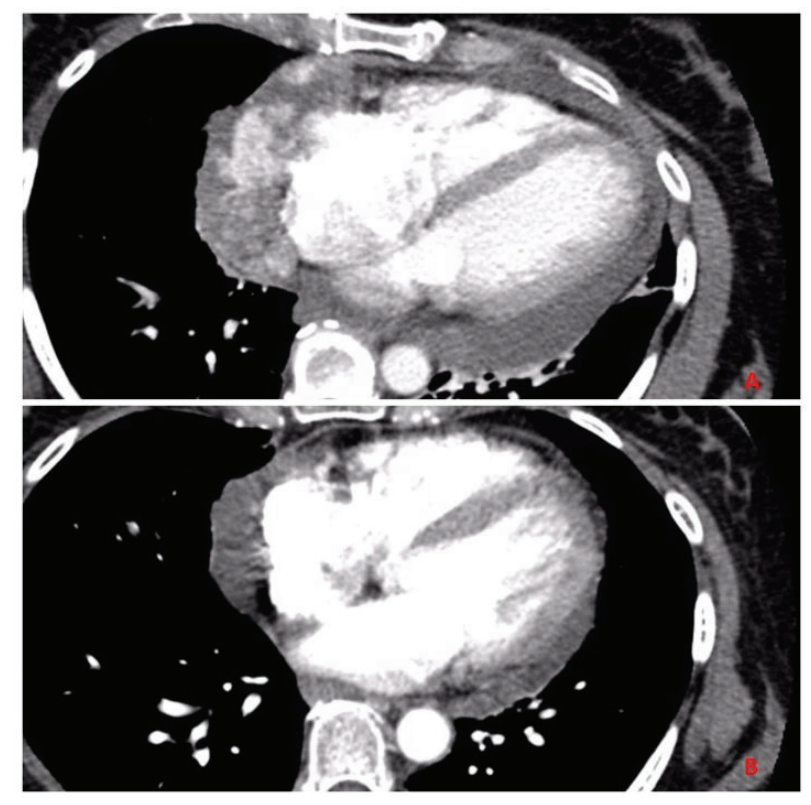

Figure 1. Panel A: CT chest with contrast before initiating chemotherapy shows invasive mass diffusely involving the pericardium and lateral right atrial wall. Panel B: CT chest with contrast after completion of chemotherapy showing partial response in the primary tumour. 


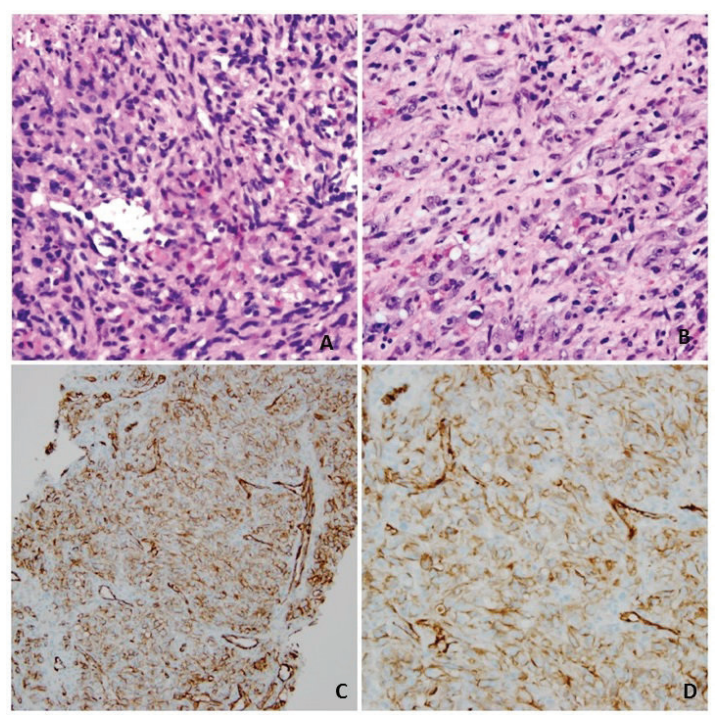

Figure 2. Panel A and B: hematoxylin-eosin stain, original magnifications $\times 20$ and $\times 40$ demonstrating spindle and epithelioid cells with prominent nucleoli, lining anastomosing, and irregular vascular channels. Panel C and D: Immunohistochemical stain for CD31 (PECAM-1), magnifications $\times 20$ and $\times 40$ showing robust staining of malignant cells lining the atypical vascular channels.

The patient had metastatic disease at the time of diagnosis. She was treated with AIM 75/10,000 regimen (doxorubicin $25 \mathrm{mg} / \mathrm{m}^{2}$ on day 1-3, ifosfamide $2,000 \mathrm{mg} / \mathrm{m}^{2}$ on day 1-5 with mesna and granulocyte colony-stimulating factor support). She completed six cycles of treatment with $25 \%$ dose reduction and minor treatment delays due to two episodes neutropenic fever. A partial response was noted after six cycles with at least $50 \%$ reduction in the tumour burden. However, within 6 weeks of completing treatment, she was diagnosed with progressive disease and opted for hospice.

\section{Discussion}

Primary cardiac sarcomas are rare and pose a diagnostic challenge to the clinician. The symptoms are varied where patients can present with congestive heart failure secondary to intracardiac obstruction, rhythm disturbances, embolisation of the tumour, chest pain, shortness of breath and/or constitutional symptoms [9]. Left and right heart sarcomas are different in presentations and characteristics. Where the left-sided tumours are more solid, less infiltrative, and tend to metastasize later; right-sided tumours are bulky, infiltrative and tend to metastasize early [10]. Imaging is critical to establish the presence of a mass, defining its mobility (useful in determining prognosis and embolic potential), presence of myocardial invasion, and location of the mass within the cardiac chambers. A three-dimensional echocardiogram (3-D Echo) is preferred to describe the tumour accurately [10]. Cardiac magnetic resonance (MR) is the best imaging technique as it provides precise spatial and contrast resolutions. Positron emission tomography (PET) is useful in establishing metastatic spread [10]. A combination of cardiac MR, 3-D Echo and PET scan is the best way to image patients with primary cardiac angiosarcoma [10]. Soft-tissue sarcoma (STS) is a heterogeneous group of disease and over 70 different molecular subtypes of are defined [11]. A detailed pathologic description and immunohistochemistry staining pattern is a must to establish the exact histologic subtype of sarcoma. Angiosarcoma typically presents as an abnormal, pleomorphic, malignant endothelial cells which express von Willebrand factor, CD34, CD31, ulex europaeus agglutinin one and vascular endothelial growth factor (VEGF) [12].

Clinical management of primary cardiac angiosarcoma involves a multimodality approach, including cardiothoracic surgery, radiation oncology and medical oncology. The median overall survival (OS) for patients with any PCT receiving surgical resection is 12 months compared to 1 month for those who do not receive any treatment [3]. Surgical resection with a microscopically negative margin (R0) is associated with the 
longest median survival of 17 months, followed by R1 resection (microscopically positive margins only) which is associated with a median survival of 6 months $[6-8,13]$. The role of adjuvant radiotherapy (RT) and chemotherapy remain controversial in the treatment of patients with cardiac sarcoma. A review of the SEER database showed that although RT was associated with a longer median OS (11 months for those receiving RT versus 4 months for those who did not receive RT), this was not a statistically significant association, especially when accounted for variability in surgical technique [3]. The authors postulated that RT should not be dismissed as a treatment modality as local control of the tumour seems to be associated with best survival outcomes [3]. The role of adjuvant chemotherapy in patients with primary cardiac sarcoma after resection remains anecdotal [14]. In an observational study of 15 patients with primary cardiac sarcoma (regardless of subtype) undergoing surgical resection, adjuvant chemotherapy resulted in an average OS of 12 months [14]. The patients received either a combination of cyclophosphamide, vincristine and dacarbazine (four patients); Ifosfamide alone (nine patients); methotrexate and vincristine (one patient) or doxorubicin alone in one patient. Even in this study, RO resection was associated with the best survival outcome (median disease-free survival (DFS) of 18 months and OS of 23.5 months). Those who had margin involvement on the tumour specimen (R1 or R2 resection) had poor DFS (4.5 months) and OS (7 months), indicating that a positive margin weighs heavily in determining the survival of a patient with PCT [13].

Neoadjuvant chemotherapy in patients with any STS is gaining momentum, especially for patients in whom surgical resection is likely to result in R1 or R2 resection [11]. In a study of 27 patients diagnosed with cardiac sarcoma (11 patients had angiosarcoma), perioperative chemotherapy (neoadjuvant, adjuvant, or both) resulted in a survival rate of $80.9 \%$ at 1 year and $61.6 \%$ at 2 years. (9) The combination of doxorubicin and Ifosfamide or gemcitabine and docetaxel or single-agent high dose ifosfamide was the chemotherapy of choice [9]. In a follow-up study, the same group reported outcomes of 44 patients with right heart sarcoma (30 patients had angiosarcoma). The findings again confirmed the OS benefit of RO resection over R1 resection (53.5 months versus 9.5 months, respectively). In addition to this, they also reported that adding neoadjuvant chemotherapy improved OS from 9.5 to 20 months [4]. They also reported that patients receiving neoadjuvant chemotherapy have a higher rate of RO resection rate; however, this observation could not achieve statistical significance. More radical and invasive measures like cardiac autotransplant followed by adjuvant chemotherapy do not result in a better survival outcome [15]. For patients with left heart sarcoma, Methodist hospital (Houston) follows the protocol of cardiac autotransplant with or without pneumonectomy, followed by adjuvant chemotherapy [16]. Despite high 30 -day mortality of $57 \%$ in the pneumonectomy group and $11 \%$ in autotransplant only group, 1-year, and 2-year survival rates are considerably high (58\% and $32 \%$, respectively). As of now, cardiac autotransplant for any PCT remains investigational.

The management of metastatic cardiac angiosarcoma relies on systemic chemotherapy. Anthracycline-based regimens are associated with a response rate of $16 \%-27 \%$ and median survival of up to 12 months [12, 17]. Taxanes (both paclitaxel and docetaxel) have shown an exceptional activity in angiosarcomas due to their anti-angiogenic properties [12]. Although the efficacy of anthracycline and taxanes in angiosarcomas have never been compared in a prospective trial, the retrospective data suggests a similar survival benefit with the two drugs. Due to the vascular nature of the tumours, early interest in anti-VEGF therapy led to phase I/II trials with bevacizumab, sunitinib, sorafenib and axitinib. Although bevacizumab showed promising activity with a $12 \%$ overall response rate, its addition to chemotherapy could not significantly improve progression-free survival compared to chemotherapy alone [12,18]. The evidence regarding the efficacy of checkpoint inhibitors in treating advanced sarcomas remains anecdotal $[19,20]$. The ALLIANCE trial data with PD-1/PDL-1 and CTLA-4 antibodies demonstrated a response rate of $5 \%-20 \%$ with similar rates of immune-related adverse events, as seen in clinical trials with other tumour types [21, 22].

\section{Conclusion}

In conclusion, primary cardiac angiosarcoma is a rare soft-tissue sarcoma associated with a high mortality rate. The clinical diagnosis is challenging for most clinicians and an accurate diagnosis requires cardiac MR, 3-D echo and the PET scan. Surgical resection with an RO margin is associated with the best outcome. Although there is a paucity of prospective trials to validate any particular approach in the treatment of cardiac angiosarcoma, there is a benefit of adding chemotherapy and radiation therapy to surgical resection. Anthracycline and taxanes are the most active cytotoxic drugs in this tumour subtype. 


\section{Funding statement}

The authors do not have any financial relations to disclose.

\section{Contribution statement}

UY wrote the manuscript. AM revised the manuscript and provided expert opinion.

\section{Conflicts of interest}

The authors do not have any conflicts of interest to declare.

\section{Patient consent}

Patient consent was obtained prior to the preparation of this report.

\section{References}

1. Reardon MJ, Malaisrie SC, and Walkes JC, et al (2006) Cardiac autotransplantation for primary cardiac tumors Ann Thorac Surg 82(2) 645-650 https://doi.org/10.1016/j.athoracsur.2006.02.086 PMID: 16863779

2. Taguchi S (2018) Comprehensive review of the epidemiology and treatments for malignant adult cardiac tumors Gen Thorac Cardiovasc Surg 66(5) 257-262 https://doi.org/10.1007/s11748-018-0912-3 PMID: 29594875

3. Hamidi M, Moody JS, and Weigel TL, et al (2010) Primary cardiac sarcoma Ann Thorac Surg 90(1) 176-181 https://doi.org/10.1016/j. athoracsur.2010.03.065 PMID: 20609770 PMCID: 4201046

4. Abu Saleh WK, Ramlawi B, and Shapira OM, et al (2017) Improved outcomes with the evolution of a neoadjuvant chemotherapy approach to right heart sarcoma Ann Thorac Surg 104(1) 90-96 https://doi.org/10.1016/j.athoracsur.2016.10.054 PMID: 28189277

5. Amano J, Nakayama J, and Yoshimura Y, et al (2013) Clinical classification of cardiovascular tumors and tumor-like lesions, and its incidences Gen Thorac Cardiovasc Surg 61(8) 435-447 https://doi.org/10.1007/s11748-013-0214-8 PMID: 23460447 PMCID: 3732772

6. Lau C, Leonard JR, and Schwann AN, et al (2018) A 20 year experience with resection of primary cardiac tumors and metastatic tumors of the heart Ann Thorac Surg

7. Truong PT, Jones SO, and Martens B, et al (2009) Treatment and outcomes in adult patients with primary cardiac sarcoma: the British Columbia Cancer Agency experience Ann Surg Oncol 16(12) 3358-3365 https://doi.org/10.1245/s10434-009-0734-8 PMID: 19830494

8. Simpson L, Kumar SK, and Okuno SH, et al (2008) Malignant primary cardiac tumors: review of a single institution experience Cancer 112(11) 2440-2446 https://doi.org/10.1002/cncr.23459 PMID: 18428209

9. Bakaeen FG, Jaroszewski DE, and Rice DC, et al (2009) Outcomes after surgical resection of cardiac sarcoma in the multimodality treatment era J Thorac Cardiovasc Surg 137(6) 1454-1460 https://doi.org/10.1016/j.jtcvs.2008.11.026 PMID: 19464464

10. Leja MJ, Shah DJ, and Reardon MJ (2011) Primary cardiac tumors Tex Heart Inst J 38(3) 261-262 PMID: 21720466 PMCID: 3113129 
11. Mangla A and Yadav U (2020) Cancer, Leiomyosarcoma (Treasure Island, FL: StatPearls)

12. Young RJ, Brown NJ, and Reed MW, et al Angiosarcoma Lancet Oncol 11(10) 983-991 PMID: 20537949

13. Mayer F, Aebert H, and Rudert M, et al (2007) Primary malignant sarcomas of the heart and great vessels in adult patients--a singlecenter experience Oncologist 12(9) 1134-1142 https://doi.org/10.1634/theoncologist.12-9-1134 PMID: 17914083

14. Llombart-Cussac A, Pivot X, Contesso G, Rhor-Alvarado A, Delord JP, Spielmann M, et al. Adjuvant chemotherapy for primary cardiac sarcomas: the IGR experience. Br J Cancer. 1998;78(12):1624-8. https://doi.org/10.1038/bjc.1998.733 PMID: 9862574 PMCID: 2063231

15. Uberfuhr P, Meiser B, and Fuchs A, et al (2002) Heart transplantation: an approach to treating primary cardiac sarcoma? J Heart Lung Transplant 21(10) 1135-1139 https://doi.org/10.1016/S1053-2498(02)00409-6 PMID: 12398881

16. Ramlawi B, Al-Jabbari O, and Blau LN, et al (2014) Autotransplantation for the resection of complex left heart tumors Ann Thorac Surg 98(3) 863-868 https://doi.org/10.1016/j.athoracsur.2014.04.125 PMID: 25086947

17. Sleijfer S, Seynaeve C, and Verweij J (2005) Using single-agent therapy in adult patients with advanced soft tissue sarcoma can still be considered standard care Oncologist 10(10) 833-841 https://doi.org/10.1634/theoncologist.10-10-833 PMID: 16314294

18. Dickson MA, D'Adamo DR, and Keohan ML, et al (2015) Phase II Trial of Gemcitabine and Docetaxel with Bevacizumab in Soft Tissue Sarcoma Sarcoma 2015532478 https://doi.org/10.1155/2015/532478 PMID: 26074722 PMCID: 4446476

19. Hofer S, Zeidler K, and Schipf A, et al (2018) Angiosarcoma of the scalp responding to nivolumab: a case report Br J Dermatol 179(2) 530-531 PMID: 29700818

20. Sindhu S, Gimber LH, and Cranmer L, et al (2017) Angiosarcoma treated successfully with anti-PD-1 therapy-a case report J Immunother Cancer 5(1) 58 https://doi.org/10.1186/s40425-017-0263-0 PMID: 28716069 PMCID: 5514460

21. D'Angelo SP, Mahoney MR, and Van Tine BA, et al (2018) Nivolumab with or without ipilimumab treatment for metastatic sarcoma (Alliance A091401): two open-label, non-comparative, randomised, phase 2 trials Lancet Oncol 19(3) 416-426 https://doi.org/10.1016/ S1470-2045(18)30006-8 PMID: 29370992 PMCID: 6126546

22. Tawbi HA, Burgess M, and Bolejack V, et al (2017) Pembrolizumab in advanced soft-tissue sarcoma and bone sarcoma (SARC028): a multicentre, two-cohort, single-arm, open-label, phase 2 trial Lancet Oncol 18(11) 1493-1501 https://doi.org/10.1016/S14702045(17)30624-1 PMID: 28988646 